\title{
Circular RNAs: New Players in Gene Regulation
}

\author{
Kalina Andreeva, Nigel G. F. Cooper* \\ Department of Anatomical Sciences and Neurobiology, University of Louisville School of Medicine, Louisville, \\ USA \\ Email: nigelcooper@louisville.edu
}

Received 27 May 2015; accepted 26 June 2015; published 29 June 2015

Copyright $@ 2015$ by authors and Scientific Research Publishing Inc.

This work is licensed under the Creative Commons Attribution International License (CC BY).

http://creativecommons.org/licenses/by/4.0/

(c) ()

\begin{abstract}
The existence of circular RNAs (circRNAs) was demonstrated over 30 years ago. They did not gain much interest at the time because they appeared to be relatively rare when compared to the abundance of the canonical linear RNAs. However, more recent evidence suggests that circRNAs are abundant in cells and tissues and possess intriguing biological properties. These recent developments have renewed our interest in this novel class of molecules. This report will provide an overview of circRNAs, discuss how they may modify our understanding of gene regulation and indicate their most likely relevance to health. The circRNAs from viruses, bacteria and archaea are not in the scope of this report, and we focused this review on circRNAs in eukaryotes.
\end{abstract}

\section{Keywords}

\section{Circular RNA, microRNA Sponges, Gene Regulation}

\section{Introduction}

Circular RNAs (circRNAs) are closed RNA loops that lack the terminating 5'-cap and 3'-polyadenylated tailstructures of linear RNAs. They are ubiquitous and have been detected in various species across kingdoms [1]-[7]. CircRNAs may consist of transcribed exons with or without introns that are scrambled (e.g. a downstream sequence is spliced to an upstream one) [8]. Due to their closed loop form, they cannot be mapped directly to the genome, which is probably one of the reasons for the late discovery of these relatively new RNA species. They appear to be ancient, conserved regulators of eukaryotic gene expression [9] and were initially detected in ribosomal depleted RNA-seq data [2]. Due to the lack of free ends circRNAs are resistant to exonucleases and have reduced rates of turnover [10]. Their lengths range between a few hundred to thousands of nuc-

${ }^{*}$ Corresponding author. 
leotides [11] and they usually contain between one and five exons [3]. The abundance of circRNAs relative to their linear RNA cousins varies between different cell types [12]. For the majority of genes from which circular and linear RNAs are produced, the amount of circRNA is typically only $1 \%$ of the linear isoforms or even less. For example, in fetal fibroblasts and HeLa cells, circular RNA compose 1\% of the RNA containing poly(A) tails [12]. However, there are reported examples where the circRNAs are more abundant than their linear counterparts [1]. Sequencing and computational analyses of RNA from different human and mouse tissues as well as various developmental stages of Caenorhabditis elegans resulted in identification of 2000 human, 1900 mouse and 700 nematode circRNAs [3]. High-throughput sequencing of libraries prepared from ribosome-depleted RNA led to the identification of significantly higher numbers $(>25,000)$ of circRNAs in human fibroblasts [1]. The analyses of 39 biological samples representing a large variety of human cell lines resulted in annotation of 7112 circRNAs [11]. In a more recent study, 29 different types or stages of mouse and human neural cells and tissues were analyzed and 15,849 mouse and 65,731 human circRNA candidates were detected [13]. Evidence for expression of circRNA was found in Drosophila [6] [12] where over 2500 circRNAs were annotated [14]. Several studies showed that different circRNA structures can be produced by alternative splicing from the same gene [9] [12], and this feature adds considerably to their diversity.

In this review, we summarize the current state of our knowledge regarding the biogenesis, regulation, and function of circRNAs and we discuss their potential uses in biomedicine.

\section{Biogenesis}

The detailed mechanisms of circRNA biogenesis have not been completely elucidated. Since the majority of circRNAs are located within protein coding gene sequences and consist of exons, it is thought that RNA polymerase II (RNA pol II) transcribes them and that their biogenesis requires the spliceosome machinery [15]. Splicing generates circRNAs via a diverse set of proposed mechanisms (Figure 1) [8].

\subsection{Back-Splicing}

The joining of a downstream 5' splice site (known as splice donor) to an upstream 3 ' splice site (known as splice

\section{Genomic DNA}

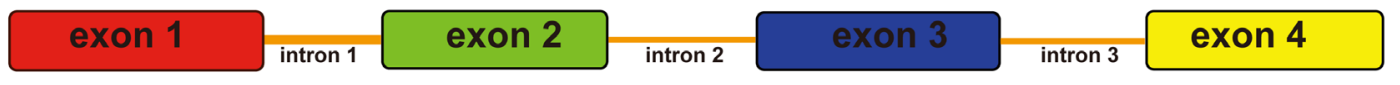

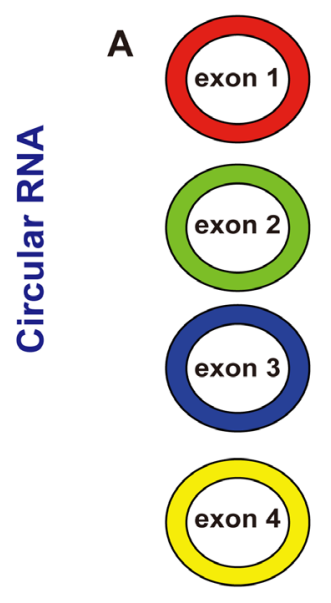

B

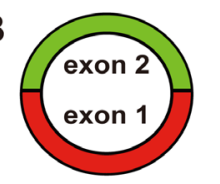

C
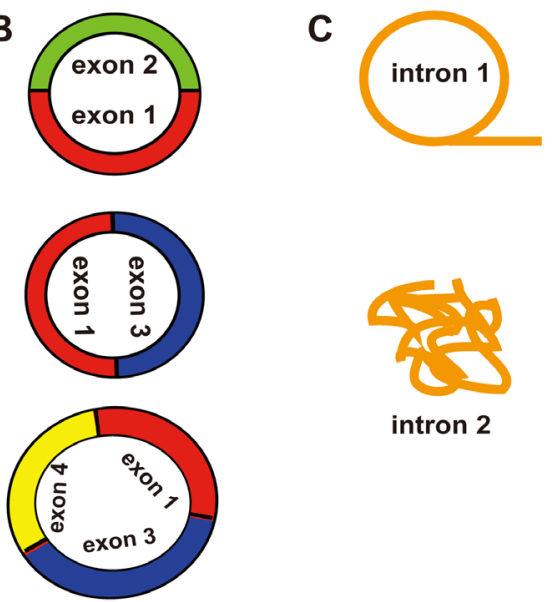
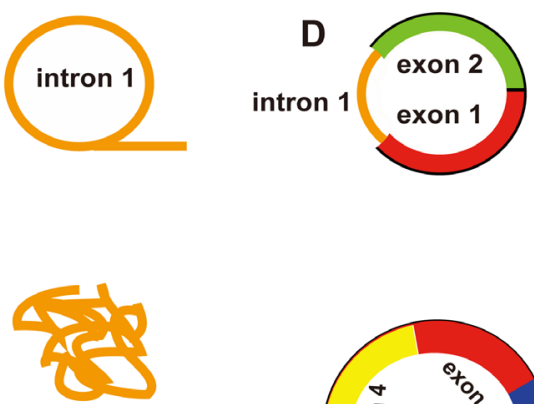

intron 2

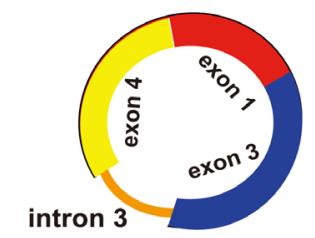

Figure 1. Circular RNAs generated by splicing. Model for how several different circular RNAs could be transcribed, in this case, from the same gene. (A) Circular RNAs can be derived from a single exon via back splicing; (B) Circular RNAs can also be formed from more than one exon via scrambled exon splicing; (C) Introns excised from precursor RNAs can also circularize to give rise to circRNAs and/or RNA lariats; (D) CircRNAs can contain introns that have been retained between one or more circularized exons. 
acceptor) on the same exon, gives rise to a circRNA and the process is referred to as "back-splicing" (Figure 1(A)). It has been demonstrated that exons could be efficiently back-spliced to generate circular mRNA and that back-splicing happened most efficiently with a relatively long exons (>1.3 kb) in human or insect cells [16].

\subsection{Scrambled Exon Splicing}

Alternatively, circRNA might be formed from more than one exon via "scrambled exon splicing" (Figure 1(B)). It has been shown that these circRNAs formed with connections between two different exons occur more frequently than between the two ends of the same exon [17].

Recent studies suggested that exon circularization depends on flanking intronic complementary sequences [18] [19] and it seems that long introns are essential for circRNA production [1] [4]. Furthermore, the introns are believed to determine the production rate of these circRNAs [15]. RNA-binding proteins appear to be important, and one RNA-binding protein, called muscleblind, was reported to promote exon circularization by binding to the flanking introns [15]. It was shown that circularized exons were often surrounded by introns that contain repeated ALU elements. The ALU elements located on both sites of the exon are in opposite orientation and through hybridization they bring together the back-splice sites to facilitate circularization [1] [19]. Interestingly, circular RNAs have also been detected in yeast where the majority of genes lack introns, and hence, the canonical alternative splicing is very rare. This observation indicated that long introns were not an absolute requirement for the production of circular RNA [9] [12]. It was shown that complementary sequences in adjacent introns could promote back-splicing, but such dsRNA structures were not necessarily a requirement [16]. A recent study demonstrated that the RNA binding protein called Quaking (QKI) is a major regulator of circRNA biogenesis during human epithelial-mesenchymal transition (EMT). QKI binds to sites flanking circRNA-forming exons and the insertion of QKI binding sites into linear RNA can induce exon circularization [20]. However, since QKI is a known regulatory factor of alternative splicing for selective mRNAs, there is skepticism regarding its specific involvement in back-splicing for the formation of circRNAs [21]. This raises the question of whether there might be proteins specifically involved in the biogenesis of circRNAs [21].

\subsection{CircRNAs from Intron Sequences}

It appears that circRNAs can be derived from intron only sequences. Excised introns might circularize and give rise to circRNA and/or RNA lariats that are splicing byproducts (Figure 1(C)). In support of this notion, 299 and 450 intronic RNAs have been detected in HeLa and H9 cells, respectively, and it has been demonstrated that many of the intronic RNAs are circular and have similar abundance to their parent mRNAs [18]. The fact that only 53 of the intronic circular RNAs were common for both cell types (HeLa and H9 cells) indicates the likelihood that these RNA species are expressed in a cell type-specific manner. In addition, it has been postulated that a consensus motif containing a $7 \mathrm{nt}$ GU-rich element near the 5' splice site, and an $11 \mathrm{nt}$ C-rich element close to the branch point, usually located 20 - 50 bp upstream of the 3' splice site, are required for the development of intronic circRNAs. It is interesting to note that circular intronic RNAs in Xenopus were reported to be passed on to their offspring via their oocytes [22]. Intriguingly, the circRNAs that derive from intron circularization have been shown to be localized to the nucleus [18], whereas the circRNAs that derive from exon back-splicing have been located in the cytoplasm [3]. This observation hints at possible differences in function for both kinds of circRNA [18]. In addition, there must be an active transport or sorting mechanism to explain the different localization of circRNAs [21]. The multitude of circRNAs recently found in the synapses of the mammalian brain [13] may provide some insights into this problem.

\subsection{CircRNAs from Exon-Intron Sequences}

Yet another type of circRNA exists [23], in which introns have been retained between circularized exons (Figure 1(D)). These circRNAs with an intron-retention property are referred to as exon-intron circRNAs, or EIcircRNAs, and like the intron only circRNAs, the EI circRNAs are also localized to the nucleus [23]. Whether these RNAs are generated co-transcriptionally or post-transcriptionally is not yet clear [23]. Taken together, studies have begun to elucidate the mode of production of circRNAs. However, their biogenesis is not completely understood and it is very likely that there are other mechanisms for generation of circRNA that remain to be discovered. The four types of circRNA listed above suggest a rich area for future research. 


\section{Regulation}

Another area of potential future research interest is related to context-related changes in expression. A number of recent studies have proposed potential mechanisms concerning regulation of circRNA abundance. For example, it seems possible that the expression of many circRNAs could be regulated during development and/or by environmental conditions. In support of this idea, circRNAs from some Schizosaccharomyces pombe genes remained stable during nitrogen starvation while others decreased relative to their corresponding linear mRNAs [9]. Numerous circRNAs have been identified in C. elegans and many of them were subjected to life cycle-dependent regulation [3]. In humans, RNA was sampled at different time points from umbilical vein endothelial cells (HUVECs) treated with tumor necrosis factor $\alpha$ (TNF $\alpha$ ) or tumor growth factor $\beta$. Different circRNAs were detected at each time point and results from these treatments indicated that CircRNA expression was condition dependent [17]. CircRNAs are present in the nervous system and are highly enriched in synapses, both in cell lines and in cultures of primary neurons [13]. They are typically up-regulated during neuronal differentiation and development [13]. Homeostatic synaptic plasticity has been induced in cultured hippocampal neurons with the aid of bicuculline, aGABAA receptor antagonist. In response to this drug, the circRNA population exhibited a dynamic behavior, resulting in up-regulation of 37 and down-regulation of 5 circRNAs [24]. These results indicated that the expression levels of the circRNAs were specifically regulated during neural plasticity. Another recent study revealed that circRNAs are subject to age-related modulation in Drosophila [14]. The study showed that the adult central nervous system expressed the highest level of circular RNAs and circRNAs continue to accumulate during aging. Therefore, circular RNAs may serve as a class of biomarker for ageing. The mechanisms underlying such age-related accumulation is not clear and the significance of such changes awaits further elucidation.

\section{Proposed Functions of circRNAs}

At present, there is limited knowledge related to the biological roles of circRNA in cells and tissues, but various functions for the circRNAs have been proposed.

\subsection{MicroRNA (miRNA) Sponges}

Some circRNAs have been described as regulatory transcripts because they can act like sponges to soak up miRNAs. Perhaps the best known example is Cerebellar Degeneration-Related protein 1 antisense, or CDR1as. It is a known circular RNA, present in both mouse and human brain. This particular circRNA contains 70 potential binding sites for miR-7, and is therefore, capable of decreasing miR-7 activity [3]. Other specific circular RNAs may function in a similar manner to regulate the activity of particular miRNAs. Thus, a circRNA transcribed from the Sry testis-specific gene in mice can absorb miR-138 [3] [5]. It has been found that circRNA generated from the ZNF91 locus (circRNA-ZNF91) contained 24 miR-23 sites as well as 39 additional sites for miR-296 [11]. ITCH is a member of the E3 ubiquitin ligases which regulate protein stability and immunological responses [25]. A recent study has revealed that cir-ITCH acted as a miRNA sponge for several different miRNAs that are associated with cancer [26]. In addition, a recent study investigated whether the predicted miRNA binding sites within human circRNA are under selective pressure, which could indicate that these binding sites are most likely functional [27]. It was found that circRNAs harbor significantly less polymorphisms at predicted miRNA target sites compared to random sites.

Although several circRNAs are predicted to serve as miRNA sponges, there may be other roles for the majority of the circRNAs. In support of this suggestion, there are circRNAs identified in yeast, for which corresponding target miRNAs have not yet been reported [9]. In addition, Salzman and colleagues (2013) analyzed thousands of circRNA and did not find enrichment of miRNAs binding sites on them [12]. Similarly, it has been shown that intronic circRNAs had little enrichment for microRNA target sites [18]. This suggests that many of the miRNA binding sites on circRNAs are functional sites under selective pressure [27]. In summary, it is plausible to propose that there are circRNAs which function as miRNA sponges but other roles have also been proposed.

\subsection{Transcriptional Regulators}

One predicted function for the circRNA relates to its positive regulation of RNA Pol II mediated transcription. 
For example, it was demonstrated that knocking down intronic circRNAs led to reduced expression of their parent genes [18]. Furthermore, one abundant circRNA from the gene ANKRD52, also known as ci-ankrd52, accumulated to its sites of transcription, where it associated with elongation Pol II machinery, and where it acted as a positive regulator of Pol II transcription. Another class of the circRNAs, namely the exon-intron circRNAs may modulate the expression of the parental genes transcriptionally and increase the levels of both circRNA and mRNA [23]. With the aid of cross-linking and immune precipitation (CLIP) experiments, 111 circRNAs have been associated with Pol II. Knockdown of two of them (circEIF3J and circPAIP2) was shown to result in a decrease in the mRNA levels of the parental genes in HeLa and HEK293 cells, respectively. Also, pull-down with either circEIF3J or circPAIP2 resulted in co-precipitation with the Pol II transcription complex [23].

\subsection{CircRNAs and Protein Production}

Twenty years ago, it was suggested that eukaryotic ribosomes can initiate translation on circRNAs which contain internal ribosome entry sites (IRES) [28]. A recent study has further examined the notion that circRNAs could function to direct protein synthesis [16]. The authors of this study engineered an IRES in a circRNA construct and detected robust protein production in transfected 293 cells. This finding indicated the possibility that some circRNAs were translated. Would they produce proteins that perhaps play a different role than the ones canonically produced? This notion has not been supported by other studies where it has not been possible to identify a naturally occurring exonic circRNA that undergoes translation [2] [12].

\subsection{Alternative Functions}

The notion that the large majority of circRNAs are unimportant byproducts of pre-mRNA splicing [11] has been challenged by several studies related to sequence conservation. For example, You and colleagues looked at conservation of circRNAs across vertebrate nervous systems and found that $23.6 \%$ of the circRNAs identified in mouse neuropil were also found in rat neuropil [24]. Numerous circRNAs were conserved not only in different cell types but also between human and mouse [3]. It has been demonstrated that the abundant circRNAs in the mammalian brain are conserved, and dynamically expressed [13]. The evidence of cellular and species conservation lends support to the notion that circRNAs have functional relevance.

There has been much progress made in this relatively new field, however, it is clear that the functions of the majority of the circRNAs remain to be elucidated [29]. Their relative abundance, evolutionary conservation and diverse cellular localizations are just the beginning of our understanding of this novel class of molecules.

\section{Health Relevance and Potential Uses in Biomedicine}

Although we are at very early stages in our understanding of the role of circRNA in the etiology of any disease, it is apparent that they have the potential for modulation of gene expression. Unlike mRNA, they are highly stable in mammalian cells. At least one specific circRNA, hsa_circ_002059, may be used as biomarker for the diagnosis of gastric carcinoma [30]. It has been demonstrated that hsa_circ_002059 levels in gastric cancerous tissues were significantly lower than those in corresponding non-tumorous tissues. A comparison of the global circRNA expression between colorectal cancer (CRC) tumor tissues and normal mucosa tissues has revealed a global reduction of circRNAs in tumor tissues from CRC patients compared to matching normal tissues [31]. The reduction was even higher in CRC cell lines. Another study has identified a circular isoform of noncoding RNA known as ANRIL (Antisense Non-coding RNA in the INK4 Locus) and it has been demonstrated that ANRIL expression correlates with susceptibility to atherosclerosis [32]. The circRNA ciRS-7/CDR1as functions as a sponge for miR-7 [3] and miR-7 is associated with a plethora of pathways and diseases (reviewed in Ref [33]). Ghosal and colleagues addressed the potential role of circRNAs in human disease by developing a very useful database (Circ2Traits) which identifies associations of circRNAs with disease/traits [34].

Recent studies have associated the overexpression of numerous miRNAs with different types of cancer. For example, miR-375 has been associated with prostate cancer progression [35], miR-25 is reported to promote gastric cancer proliferation, invasion, and migration [36], miR-145 and miR-378* were linked to the probability of colorectal cancer [37], and miR-221/222 was reported to belinked to the progression of pancreatic cancer [38]. Therefore, the absorption of such overexpressed miRNAs by synthetic circRNAs should be investigated for their potential as cancer suppressors. Studies describing a new sponge technology indicated that circRNAs can be ef- 
fective in de-repression of miRNA target genes in cellular systems [39]-[41]. In one such study, Kluiver and colleagues have developed a fast and efficient method to generate sponges that can be applied to inhibit one or more miRNAs. These investigators inserted a SanDI restriction enzyme site in the pMSCV-PIG vector, which resulted in a new vector (pMSCV-PIG-sp) containing non-palindromic overhangs. In the process of ligating oligo duplexes with SanDI compatible ends with SanDI digested pMSCV-PIG-sp, sponge constructs with a variable number of miRNA binding sites can be generated in a single ligation reaction. Using different vector to oligo duplex ratios, the number of the miRNA binding sites can be controlled. The authors used several different approaches to demonstrate that the sponges are efficient inhibitors of miRNAs [39]. They performed luciferase assays to confirm that miRNAs can bind to the sponges in a dose dependent manner. Further, Green Fluorescent Protein (GFP) assays were used to show that the sponges functionally inhibit miRNAs. Finally, AGO2 immunoprecipitation (AGO2-IP) assays were used to demonstrate that the sponges were enriched in the AGO2-IP fraction [39]. Given that miRNAs have been implicated not only in cancer but in numerous neurological [42][44], cardiovascular [45]-[47] and autoimmune diseases [48]-[50], the miRNA sponges could be another useful tool in the arsenal for combatting disease.

\section{Perspectives and Future Directions}

CircRNAs represent an exciting area of focus for future discoveries. However, many areas in this field remain largely unexplored. The regulation, abundance and turnover of circRNAs remain to be more completely investigated. This might be partially due to the observation that circRNAs have been largely undetected due to their lack of free ends. The recent findings that circRNAs are reduced in tumor tissues [31] and that their expression correlates with susceptibility to atherosclerosis [32] suggest that the levels of circRNAs can be regulated through both biosynthetic and decay processes.

Another possible avenue for future research is the identification of mechanisms by which the spliceosome selects particular exons and not others to circularize. In addition, it is largely unknown if the circularization occurs co-transcriptionally or post-transcriptionally. Hypothetically, every exon that has splicing signals on both of its ends (5'- and 3'-end) can circularize, however this is not the case. There could be several exons that circularize in the same circle (Figure 1(B)), and in some cases the introns between them have been maintained (Figure $1(D))$. The choice between the different modes of splicing must be tightly regulated since this could change the localization and/or the function of the circRNAs.

Given that some protein coding genes yield many more circRNA than linear RNA isoforms [1] [4], one can argue that the function of some genes is to give rise to circRNA instead of proteins. In this scenario the functional output of a gene would change fundamentally. This might be an intriguing, if somewhat speculative, avenue for future research.

One exciting area for future exploration would be to search for the presence of circRNAs in body fluids. It has been shown that circRNAs are resistant to degradation by the RNA decay mechanisms which typically recognize the ends of linear RNAs, and therefore circRNAs could have long half-lives. It is possible that circRNA possess remarkable stability in the blood stream and/or other bodily fluids. The presence of altered circRNA expression patterns in the fluids might be the cause or the result of various disease states. In line with this, there is one recent study that reports the presence of 400 circRNAs in human cell free saliva (CFC) [51]. Stable biomarkers in saliva are of great interest due to the possibility of noninvasive detection methods for the presence of diseases.

\section{Conclusion}

In summary, circRNAs add a new level of complexity to the narrative of our transcriptome. CircRNAs possess the potential to regulate protein coding and non-coding sequences. This potential together with the detection of ever growing numbers of circRNAs and their demonstrated conservation across species, provide support for their functional relevance. We look forward with excitement to the future studies which will uncover the full biological relevance of circRNAs and the implications in health and disease.

\section{Conflict of Interest Statement}

The authors declare no conflict of interest. 


\section{Support}

This work was supported in part by grants from the National Eye Institute R01EY017594, and the National Institute of General Medical Sciences P20 GM103436.

\section{References}

[1] Jeck, W.R., Sorrentino, J.A., Wang, K., Slevin, M.K., Burd, C.E., Liu, J., Marzluff, W.F. and Sharpless, N.E. (2013) Circular RNAs Are Abundant, Conserved, and Associated with ALU Repeats. RNA, 19, 141-157. http://dx.doi.org/10.1261/rna.035667.112

[2] Jeck, W.R. and Sharpless, N.E. (2014) Detecting and Characterizing Circular RNAs. Nature Biotechnology, 32, 453-461. http://dx.doi.org/10.1038/nbt.2890

[3] Memczak, S., Jens, M., Elefsinioti, A., Torti, F., Krueger, J., Rybak, A., Maier, L., Mackowiak, S.D., Gregersen, L.H., Munschauer, M., et al. (2013) Circular RNAs Are a Large Class of Animal RNAs with Regulatory Potency. Nature, 495, 333-338. http://dx.doi.org/10.1038/nature11928

[4] Salzman, J., Gawad, C., Wang, P.L., Lacayo, N. and Brown, P.O. (2012) Circular RNAs Are the Predominant Transcript Isoform from Hundreds of Human Genes in Diverse Cell Types. PLoS One, 7, e30733. http://dx.doi.org/10.1371/journal.pone.0030733

[5] Hansen, T.B., Jensen, T.I., Clausen, B.H., Bramsen, J.B., Finsen, B., Damgaard, C.K. and Kjems, J. (2013) Natural RNA Circles Function as Efficient microRNA Sponges. Nature, 495, 384-388. http://dx.doi.org/10.1038/nature11993

[6] Houseley, J.M., Garcia-Casado, Z., Pascual, M., Paricio, N., O’Dell, K.M., Monckton, D.G. and Artero, R.D. (2006) Noncanonical RNAs from Transcripts of the Drosophila muscleblind Gene. The Journal of Heredity, 97, 253-260. http://dx.doi.org/10.1093/jhered/esj037

[7] Doose, G., Alexis, M., Kirsch, R., Findeiss, S., Langenberger, D., Machne, R., Morl, M., Hoffmann, S. and Stadler, P.F. (2013) Mapping the RNA-Seq Trash Bin: Unusual Transcripts in Prokaryotic Transcriptome Sequencing Data. RNA Biology, 10, 1204-1210. http://dx.doi.org/10.4161/rna.24972

[8] Vicens, Q. and Westhof, E. (2014) Biogenesis of Circular RNAs. Cell, 159, 13-14. http://dx.doi.org/10.1016/j.cell.2014.09.005

[9] Wang, P.L., Bao, Y., Yee, M.C., Barrett, S.P., Hogan, G.J., Olsen, M.N., Dinneny, J.R., Brown, P.O. and Salzman, J. (2014) Circular RNA Is Expressed across the Eukaryotic Tree of Life. PLoS One, 9, e95116. http://dx.doi.org/10.1371/journal.pone.0090859

[10] Suzuki, H., Zuo, Y., Wang, J., Zhang, M.Q., Malhotra, A. and Mayeda, A. (2006) Characterization of RNase R-Digested Cellular RNA Source That Consists of Lariat and Circular RNAs from Pre-mRNA Splicing. Nucleic Acids Research, 34, e63. http://dx.doi.org/10.1093/nar/gkl151

[11] Guo, J.U., Agarwal, V., Guo, H. and Bartel, D.P. (2014) Expanded Identification and Characterization of Mammalian Circular RNAs. Genome Biology, 15, 409. http://dx.doi.org/10.1186/s13059-014-0409-z

[12] Salzman, J., Chen, R.E., Olsen, M.N., Wang, P.L. and Brown, P.O. (2013) Cell-Type Specific Features of Circular RNA Expression. PLoS Genetics, 9, e1003777. http://dx.doi.org/10.1371/journal.pgen.1003777

[13] Rybak-Wolf, A., Stottmeister, C., Glazar, P., Jens, M., Pino, N., Giusti, S., Hanan, M., Behm, M., Bartok, O., Ashwal-Fluss, R., et al. (2015) Circular RNAs in the Mammalian Brain Are Highly Abundant, Conserved, and Dynamically Expressed. Molecular Cell, 58, 870-885. http://dx.doi.org/10.1016/j.molcel.2015.03.027

[14] Westholm, J.O., Miura, P., Olson, S., Shenker, S., Joseph, B., Sanfilippo, P., Celniker, S.E., Graveley, B.R. and Lai, E.C. (2014) Genome-Wide Analysis of Drosophila Circular RNAs Reveals Their Structural and Sequence Properties and Age-Dependent Neural Accumulation. Cell Reports, 9, 1966-1980. http://dx.doi.org/10.1016/j.celrep.2014.10.062

[15] Ashwal-Fluss, R., Meyer, M., Pamudurti, N.R., Ivanov, A., Bartok, O., Hanan, M., Evantal, N., Memczak, S., Rajewsky, N. and Kadener, S. (2014) circRNA Biogenesis Competes with Pre-mRNA Splicing. Molecular Cell, 56, 5566. http://dx.doi.org/10.1016/j.molcel.2014.08.019

[16] Wang, Y. and Wang, Z. (2015) Efficient Backsplicing Produces Translatable Circular mRNAs. RNA, 21, 172-179. http://dx.doi.org/10.1261/rna.048272.114

[17] Kelly, S., Greenman, C., Cook, P.R. and Papantonis, A. (2015) Exon Skipping Is Correlated with Exon Circularization. Journal of Molecular Biology, in press. http://dx.doi.org/10.1016/j.jmb.2015.02.018

[18] Zhang, Y., Zhang, X.O., Chen, T., Xiang, J.F., Yin, Q.F., Xing, Y.H., Zhu, S., Yang, L. and Chen, L.L. (2013) Circular Intronic Long Noncoding RNAs. Molecular Cell, 51, 792-806. http://dx.doi.org/10.1016/j.molcel.2013.08.017

[19] Liang, D. and Wilusz, J.E. (2014) Short Intronic Repeat Sequences Facilitate Circular RNA Production. Genes \& Development, 28, 2233-2247. http://dx.doi.org/10.1101/gad.251926.114 
[20] Conn, S.J., Pillman, K.A., Toubia, J., Conn, V.M., Salmanidis, M., Phillips, C.A., Roslan, S., Schreiber, A.W., Gregory, P.A. and Goodall, G.J. (2015) The RNA Binding Protein Quaking Regulates Formation of circRNAs. Cell, 160, 11251134. http://dx.doi.org/10.1016/j.cell.2015.02.014

[21] Chen, L. and Shan, G. (2015) Circular RNAs Remain Peculiarly Unclear in Biogenesis and Function. Science China Life Sciences, in press. http://dx.doi.org/10.1007/s11427-015-4855-y

[22] Gaelle, J.S., Talhouarne, G.J. and Gall, J.G. (2014) Lariat Intronic RNAs in the Cytoplasm of Xenopus tropicalis Oocytes. RNA, 20, 1476-1487. http://dx.doi.org/10.1261/rna.045781.114

[23] Li, Z., Huang, C., Bao, C., Chen, L., Lin, M., Wang, X., Zhong, G., Yu, B., Hu, W., Dai, L., et al. (2015) Exon-Intron Circular RNAs Regulate Transcription in the Nucleus. Nature Structural \& Molecular Biology, 22, 256-264. http://dx.doi.org/10.1038/nsmb.2959

[24] You, X., Vlatkovic, I., Babic, A., Will, T., Epstein, I., Tushev, G., Akbalik, G., Wang, M., Glock, C., Quedenau, C., et al. (2015) Neural Circular RNAs Are Derived from Synaptic Genes and Regulated by Development and Plasticity. Nature Neuroscience, 18, 603-610. http://dx.doi.org/10.1038/nn.3975

[25] Rossi, M., Inoue, S., Walewska, R., Knight, R.A., Dyer, M.J., Cohen, G.M. and Melino, G. (2009) Caspase Cleavage of Itch in Chronic Lymphocytic Leukemia Cells. Biochemical and Biophysical Research Communications, 379, 659664. http://dx.doi.org/10.1016/j.bbrc.2008.11.154

[26] Li, F., Zhang, L., Li, W., Deng, J., Zheng, J., An, M., Lu, J. and Zhou, Y. (2015) Circular RNA ITCH Has Inhibitory Effect on ESCC by Suppressing the Wnt/Beta-Catenin Pathway. Oncotarget, 6, 6001-6013.

[27] Thomas, L.F. and Saetrom, P. (2014) Circular RNAs Are Depleted of Polymorphisms at microRNA Binding Sites. Bioinformatics (Oxford, England), 30, 2243-2246. http://dx.doi.org/10.1093/bioinformatics/btu257

[28] Chen, C.Y. and Sarnow, P. (1995) Initiation of Protein Synthesis by the Eukaryotic Translational Apparatus on Circular RNAs. Science, 268, 415-417. http://dx.doi.org/10.1126/science.7536344

[29] Wilusz, J.E. and Sharp, P.A. (2013) Molecular Biology. A Circuitous Route to Noncoding RNA. Science, 340, $440-441$. http://dx.doi.org/10.1126/science.1238522

[30] Li, P., Chen, S., Chen, H., Mo, X., Li, T., Shao, Y., Xiao, B. and Guo, J. (2015) Using Circular RNA as a Novel Type of Biomarker in the Screening of Gastric Cancer. Clinica Chimica Acta, 444, 132-136. http://dx.doi.org/10.1016/j.cca.2015.02.018

[31] Bachmayr-Heyda, A., Reiner, A.T., Auer, K., Sukhbaatar, N., Aust, S., Bachleitner-Hofmann, T., Mesteri, I., Grunt, T.W., Zeillinger, R. and Pils, D. (2015) Correlation of Circular RNA Abundance with Proliferation—Exemplified with Colorectal and Ovarian Cancer, Idiopathic Lung Fibrosis, and Normal Human Tissues. Scientific Reports, 5, Article No. 8057. http://dx.doi.org/10.1038/srep08057

[32] Burd, C.E., Jeck, W.R., Liu, Y., Sanoff, H.K., Wang, Z. and Sharpless, N.E. (2010) Expression of Linear and Novel Circular Forms of an INK4/ARF-Associated Non-Coding RNA Correlates with Atherosclerosis Risk. PLoS Genetics, 6, e1001233. http://dx.doi.org/10.1371/journal.pgen.1001233

[33] Hansen, T.B., Kjems, J. and Damgaard, C.K. (2013) Circular RNA and miR-7 in Cancer. Cancer Research, 73, 56095612. http://dx.doi.org/10.1158/0008-5472.CAN-13-1568

[34] Ghosal, S., Das, S., Sen, R., Basak, P. and Chakrabarti, J. (2013) Circ2Traits: A Comprehensive Database for Circular RNA Potentially Associated with Disease and Traits. Frontiers in Genetics, 4, 283. http://dx.doi.org/10.3389/fgene.2013.00283

[35] Costa-Pinheiro, P., Ramalho-Carvalho, J., Vieira, F.Q., Torres-Ferreira, J., Oliveira, J., Goncalves, C.S., Costa, B.M., Henrique, R. and Jeronimo, C. (2015) MicroRNA-375 Plays a Dual Role in Prostate Carcinogenesis. Clinical Epigenetics, 7, 42. http://dx.doi.org/10.1186/s13148-015-0076-2

[36] Gong, J., Cui, Z., Li, L., Ma, Q., Wang, Q., Gao, Y. and Sun, H. (2015) MicroRNA-25 Promotes Gastric Cancer Proliferation, Invasion, and Migration by Directly Targeting F-Box and WD-40 Domain Protein 7, FBXW7. Tumor Biology, in press. http://dx.doi.org/10.1007/s13277-015-3510-3

[37] Peng, J., Xie, Z., Cheng, L., Zhang, Y., Chen, J., Yu, H., Li, Z. and Kang, H. (2015) Paired Design Study by Real-Time PCR: miR-378* and miR-145 Are Potent Early Diagnostic Biomarkers of Human Colorectal Cancer. BMC Cancer, 15, 158. http://dx.doi.org/10.1186/s12885-015-1123-2

[38] Xu, Q., Li, P., Chen, X., Zong, L., Jiang, Z., Nan, L., Lei, J., Duan, W., Zhang, D., Li, X., et al. (2015) miR-221/222 Induces Pancreatic Cancer Progression through the Regulation of Matrix Metalloproteinases. Oncotarget, in press.

[39] Kluiver, J., Gibcus, J.H., Hettinga, C., Adema, A., Richter, M.K., Halsema, N., Slezak-Prochazka, I., Ding, Y., Kroesen, B.J. and van den Berg, A. (2012) Rapid Generation of microRNA Sponges for microRNA Inhibition. PLoS ONE, 7, e29275. http://dx.doi.org/10.1371/journal.pone.0029275

[40] Ebert, M.S., Neilson, J.R. and Sharp, P.A. (2007) MicroRNA Sponges: Competitive Inhibitors of Small RNAs in 
Mammalian Cells. Nature Methods, 4, 721-726. http://dx.doi.org/10.1038/nmeth1079

[41] Gentner, B., Schira, G., Giustacchini, A., Amendola, M., Brown, B.D., Ponzoni, M. and Naldini, L. (2009) Stable Knockdown of microRNA in Vivo by Lentiviral Vectors. Nature Methods, 6, 63-66. http://dx.doi.org/10.1038/nmeth.1277

[42] Chen, W. and Qin, C. (2015) General Hallmarks of microRNAs in Brain Evolution and Development. RNA Biology, in Press.

[43] Paschon, V., Takada, S.H., Ikebara, J.M., Sousa, E., Raeisossadati, R., Ulrich, H. and Kihara, A.H. (2015) Interplay between Exosomes, microRNAs and Toll-Like Receptors in Brain Disorders. Molecular Neurobiology, in press. http://dx.doi.org/10.1007/s12035-015-9142-1

[44] Luo, T., Yin, S., Shi, R., Xu, C., Wang, Y., Cai, J., Yue, Y. and Wu, A. (2015) miRNA Expression Profile and Involvement of Let-7d-APP in Aged Rats with Isoflurane-Induced Learning and Memory Impairment. PLoS ONE, 10, e0119336. http://dx.doi.org/10.1371/journal.pone.0119336

[45] Liu, Y., Chen, S., Zhang, J., Shan, S., Chen, L., Wang, R., Kan, J. and Xu, T. (2015) Analysis of Serum MicroRNAs as Potential Biomarker in Coronary Bifurcation Lesion. Disease Markers, 2015, Article ID: 351015.

[46] Dorn II, G.W. (2015) Great Expectations: MicroRNA-30d and Cardiac Resynchronization Therapy. Circulation, in press. http://dx.doi.org/10.1161/CIRCULATIONAHA.115.017176

[47] Das, S. and Halushka, M.K. (2015) Extracellular Vesicle microRNA Transfer in Cardiovascular Disease. Cardiovascular pathology: The Official Journal of the Society for Cardiovascular Pathology, 24, 199-206.

[48] Naghavian, R., Ghaedi, K., Kiani-Esfahani, A., Ganjalikhani-Hakemi, M., Etemadifar, M. and Nasr-Esfahani, M.H. (2015) miR-141 and miR-200a, Revelation of New Possible Players in Modulation of Th17/Treg Differentiation and Pathogenesis of Multiple Sclerosis. PLoS ONE, 10, e0124555. http://dx.doi.org/10.1371/journal.pone.0124555

[49] Schaefer, J.S., Attumi, T., Opekun, A.R., Abraham, B., Hou, J., Shelby, H., Graham, D.Y., Streckfus, C. and Klein, J.R. (2015) MicroRNA Signatures Differentiate Crohn’s Disease from Ulcerative Colitis. BMC Immunology, $16,5$. http://dx.doi.org/10.1186/s12865-015-0069-0

[50] Tili, E., Michaille, J.J., Costinean, S. and Croce, C.M. (2008) MicroRNAs, the Immune System and Rheumatic Disease. Nature Clinical Practice Rheumatology, 4, 534-541. http://dx.doi.org/10.1038/ncprheum0885

[51] Bahn, J.H., Zhang, Q., Li, F., Chan, T.M., Lin, X., Kim, Y., Wong, D.T. and Xiao, X. (2015) The Landscape of microRNA, Piwi-Interacting RNA, and Circular RNA in Human Saliva. Clinical Chemistry, 61, 221-230.

http://dx.doi.org/10.1373/clinchem.2014.230433 